\title{
Hidden Symmetries, Instabilities, and Current Suppression in Brownian Ratchets
}

\author{
David Cubero $^{1, *}$ and Ferruccio Renzoni ${ }^{2,+}$ \\ ${ }^{1}$ Departamento de Física Aplicada I, EUP, Universidad de Sevilla, Calle Virgen de África 7, 41011 Sevilla, Spain \\ ${ }^{2}$ Department of Physics and Astronomy, University College London, Gower Street, London WC1E 6BT, United Kingdom
}

(Received 12 September 2015; published 7 January 2016)

\begin{abstract}
The operation of Brownian motors is usually described in terms of out-of-equilibrium and symmetrybreaking settings, with the relevant spatiotemporal symmetries identified from the analysis of the equations of motion for the system at hand. When the appropriate conditions are satisfied, symmetry-related trajectories with opposite current are thought to balance each other, yielding suppression of transport. The direction of the current can be precisely controlled around these symmetry points by finely tuning the driving parameters. Here we demonstrate, by studying a prototypical Brownian ratchet system, the existence of hidden symmetries, which escape identification by the standard symmetry analysis, and which require different theoretical tools for their revelation. Furthermore, we show that system instabilities may lead to spontaneous symmetry breaking with unexpected generation of directed transport.
\end{abstract}

DOI: 10.1103/PhysRevLett.116.010602

Motion at the nanoscale presents features very different from those encountered in the macroscopic world. Noise is a dominant process at such a scale, and may contribute constructively to the dynamics rather than playing the usual role of a disturbance. New mechanisms of transport emerge at the nanoscale; in particular, directed motion may occur in the absence of an applied bias force. Brownian ratchets [1-3], the archetypal model system capturing the mechanisms behind such a transport process, represent a key to understanding several biological processes [4,5]; they also have inspired a plethora of new nanodevices displaying directed motion [6-21]. All these systems are usually described in terms of operation away from thermal equilibrium, with directed motion following from the breaking of certain spatiotemporal symmetries, which are identified from the analysis of the equations of motion for the system at hand. Here we prove the existence of hidden symmetries, which escape identification by the standard symmetry analysis $[2,3,22,23]$, and require different theoretical tools for their revelation. The main assumption of the standard symmetry analysis-i.e., that two trajectories connected by a symmetry transformation carry the same statistical weight, a reasoning that can be traced back to Loschmidt's paradox [24]—yields incorrect predictions in these dissipative systems, failing to account for system instabilities that lead to spontaneous symmetry breaking.

Results.-A large class of Brownian motor systems, which includes particles in solution [8], vortices in superconductors [14], and atoms in dissipative optical lattices [21], corresponds to a Brownian particle diffusing in a periodic potential under the action of a driving force with zero average. The particle's motion is described by the following Langevin equation:

$$
m \ddot{x}=-\gamma \dot{x}+\mathcal{F}(x, t)+\xi(t)
$$

where $\gamma$ is the friction coefficient, $\mathcal{F}(x, t)$ is a generic deterministic force, and $\xi(t)$ is a fluctuating force, modeled as a Gaussian white noise with autocorrelation $\left\langle\xi(t) \xi\left(t^{\prime}\right)\right\rangle=2 \Gamma \delta\left(t-t^{\prime}\right)$, with the noise strength $\Gamma$ related to the temperature $T$ of the environment via the fluctuationdissipation relation $\Gamma=\gamma k_{B} T$. The directed current is defined as $\langle v\rangle=\lim _{t \rightarrow \infty}\langle x(t)\rangle / t$, where the angle brackets denote the average over noise realizations. For finite noise strengths $\Gamma>0$, ergodicity implies $\langle v\rangle=\lim _{t \rightarrow \infty} x(t) / t$. In very small systems, from the nanoscale to the microscale, the Brownian dynamics of small particles is frequently in the overdamped regime, where inertia effects-the term $m \ddot{x}$ in (1) — can be neglected. This is the regime of interest here.

The standard symmetry analysis $[2,3,22,23]$ relies on the identification of transformations that leave the equation of motion (1) unchanged and reverse the sign of the particle momentum. Trajectories with opposite momentum are equivalent, with a net null contribution to the directed current, which thus turns out to be zero. We will show that this picture does not fully capture the basic principles behind the operation of Brownian ratchets. To do this, we consider a more general approach [25], and regard the directed current $\langle v\rangle$ as a generic functional of the driving force $\mathcal{F}$, thus using the notation $v[\mathcal{F}(x, t)]$. Several properties follow from symmetry considerations.

First, due to the vectorial nature of both the force $\mathcal{F}$ and the current, the transformation $x \rightarrow-x$ yields the following property:

$$
v[-\mathcal{F}(-x, t)]=-v[\mathcal{F}(x, t)] .
$$

Second, an arbitrary translation along the $x$ or $t$ axis does not alter the current, i.e.,

$$
v[\mathcal{F}(x, t)]=v\left[\mathcal{F}\left(x+x_{0}, t\right)\right]=v\left[\mathcal{F}\left(x, t+t_{0}\right)\right] .
$$


Let us consider now a forced ratchet, i.e., $\mathcal{F}(x, t)=$ $f(x)+F(t)$, where $f(x)=-\partial U(x) / \partial x$ is a conservative force and $F(t)$ is a driving force.

If the system is spatially symmetric with respect to a certain point $x_{0}$, then the potential satisfies $U\left(x+x_{0}\right)=$ $U\left(-x+x_{0}\right)$. Without loss of generality, we choose the coordinate's origin such that $x_{0}=0$. Then $-f(-x)=f(x)$, which together with (2) yields a characteristic property of spatially symmetric systems,

$$
v[f(x)-F(t)]=-v[f(x)+F(t)] .
$$

A shift-symmetric force is defined as $F\left(t+t_{0}\right)=-F(t)-$ for periodic drives $t_{0}=\tau / 2$, where $\tau$ is the period, $F(t+\tau)=F(t)$. The direct application of properties (4) and (3) yields no current for shift-symmetric forces in spatially symmetric systems,

$$
\begin{aligned}
v[f(x)-F(t)] & =v\left[f(x)+F\left(t+t_{0}\right)\right]=v[f(x)+F(t)] \\
& =-v[f(x)+F(t)] .
\end{aligned}
$$

This is a well-known result of spatially symmetric systems, already captured by the standard symmetry analysis $[2,3,22,23]$. However, our current approach reveals two additional symmetries for overdamped one-dimensional systems, which are not captured by the standard approach. They are

$$
\begin{aligned}
v[f(-x)+F(t)] & =v[f(x)+F(t)], \\
v[f(x)+F(-t)] & =v[f(x)+F(t)] .
\end{aligned}
$$

A proof of (6) and (7) based on the Smoluchowski equation is given in the Supplemental Material [26]. The symmetries (6) and (2), together with (3), yield the following property for shift-symmetric potentials:

$$
\begin{aligned}
v[f(x)+F(t)] & =v[f(-x)+F(t)]=-v[-f(x)-F(t)] \\
& =-v[f(x+L / 2)-F(t)]=-v[f(x)-F(t)] .
\end{aligned}
$$

This is the same property as Eq. (4) and, proceeding as before, it implies current suppression when combined with a shift-symmetric driving force. Therefore, quite counterintuitively, in one-dimensional overdamped systems, the condition for current suppression for systems with shiftsymmetric potentials — like the one shown in Fig. 1(b) - is, despite being spatially asymmetric, the same as for spatially symmetric potentials. Figure 1(c) confirms this unexpected behavior for large enough frictions. In the underdamped regime this property is not satisfied exactly. Nevertheless, even in this regime the overdamped symmetry (6) identified here has a lasting effect: The zero-current point determined by the overdamped symmetry is displaced to a lower value

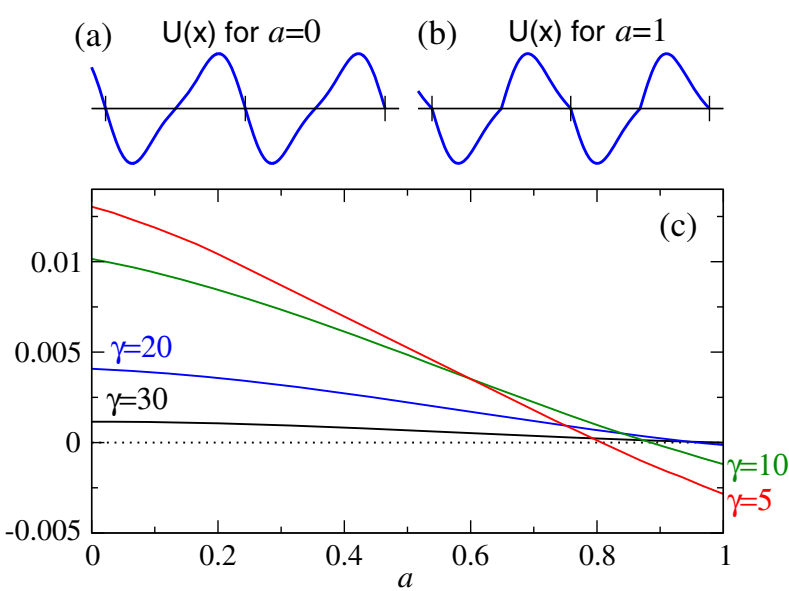

FIG. 1. Shift-symmetric potentials act like spatially symmetric ones in one-dimensional overdamped systems. (a) Ratchet potential $U_{\text {rat }}(x)=-U_{0}[\sin (k x)+(1 / 4) \sin (2 k x)]$ with period $L=2 \pi / k$. (b) Shift-symmetric potential defined from $U_{\text {rat }}(x)$ as $U_{\mathrm{ss}}(x)=U_{\text {rat }}(x)$ in the first half-period, and $U_{\mathrm{ss}}(x)=$ $-U_{\mathrm{ss}}(x-L / 2)$ in the second half-period. (c) Directed current for a Brownian particle subject to the mixed potential $U(x)=$ $U_{\text {rat }}(x)(1-a)+U_{\mathrm{ss}}(x) a$ and to a shift-symmetric force defined by $F(t)=g(t) \equiv A[\sin (\omega t)+(1 / 4) \sin (2 \omega t)]$ in the first halfperiod, and $F(t)=-g(t-\tau / 2)$ in the second half, where $\tau=2 \pi / \omega$. Reduced units are defined such as $m=L=10 \omega=1$. Other parameters are $A=4, U_{0}=10 / 2 \pi$, and $\Gamma=10$. The directed current vanishes in the overdamped limit (large frictions $\gamma)$ for the shift-symmetric potential $(a=1)$ because of hidden symmetries.

of the symmetry parameter $a$ in the underdamped regime. Thus, the overdamped symmetry (6) determines a current reversal in the underdamped regime.

The discovery of hidden symmetries reported above does not represent the only departure from the conclusions that can be drawn from the standard symmetry analysis. The presence of instabilities may also alter the picture, as trajectories that are solutions of the equations of motion with opposite momenta may have very different stability properties, and, thus, result into a total nonzero contribution to the system current. Such a scenario of spontaneous symmetry breaking is best illustrated via a specific case study.

In the overdamped regime, from every solution $x(t)$, the trajectory $\tilde{x}(t)=x(-t)+L / 2$ is also a solution of (1) provided the potential is shift symmetric. It corresponds to a transformed random force $\tilde{\xi}(t)=-\xi(-t)$, which is statistically equivalent to $\xi(t)$, and a driving force $\tilde{F}(t)=-F(-t)$. Following the standard symmetry analysis, no current is expected when antisymmetric driving forces $F\left(t+t_{0}\right)=-F\left(-t+t_{0}\right)$ are applied [3,22,23,41]an appropriate choice of the time origin yields $t_{0}=0$. This prediction is correct in one-dimensional systems, as readily verified by numerical simulations. However, the same reasoning also predicts no current in the case of higher dimensions, a result that is contradicted by our numerical 


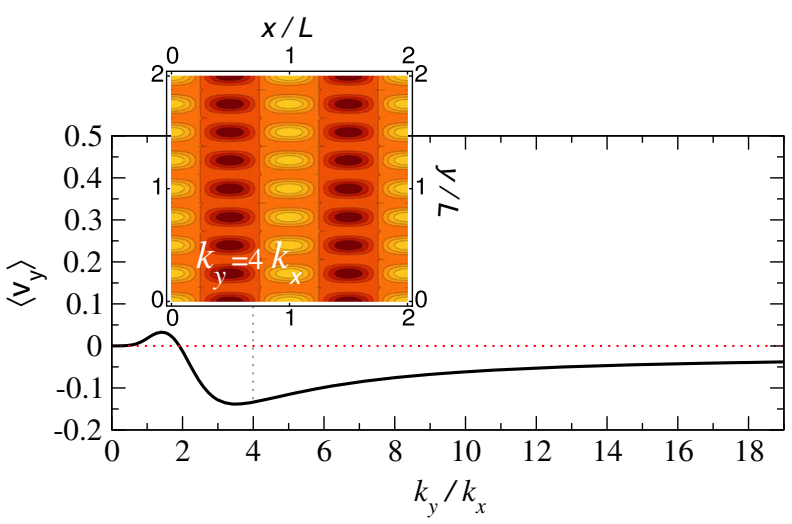

FIG. 2. Breaking of symmetries (6), (7) in a 2D overdamped system. The driving force is $\mathbf{F}(t)=A\left[\cos (\omega t) \mathbf{e}_{x}+\right.$ $\cos (2 \omega t+\pi / 2) \mathbf{e}_{y}$ ], i.e., a biharmonic drive split [42] in two perpendicular directions. The potential is $U(x, y)=$ $U_{0} \cos \left(k_{x} x\right)\left[1+\cos \left(k_{y} y\right)\right]$, which is spatially symmetric in both directions, and shift symmetric along the $x$ direction. The current is produced in the $y$ direction only-due to the symmetry (5) in the $x$ direction - through the coupling with the dynamics in the $x$ direction. Though the driving force is antisymmetric, a nonzero current is observed when $k_{x}$ and $k_{y}$ are comparable. Reduced units are defined such that $m=k_{x}=\omega=1$. Other parameters are $U_{0}=\gamma=50, A=2 \gamma$, and $\Gamma=0.1 \gamma^{2}$. The inset illustrates the potential landscape for $k_{y}=4 k_{x}$, with $L=2 \pi / k_{x}$.

simulations, as shown in Fig. 2 for a two-dimensional potential and an applied split biharmonic drive, as well as by independent results by Reimann's group (see Ref. [43], p. 16). The presence of instabilities is the key to understanding such an unexpected, spontaneous symmetrybreaking behavior. The standard analysis fails to account for the actual instability of the transformed solutions $\tilde{x}(t)$, which makes them very unlikely. Even in the noiseless limit, the above transformation maps stable oscillations about the potential minima into highly unstable oscillations about potential maxima [44]. We have verified via numerical simulations that, given a stable solution $x(t)$, the transformed solution $\tilde{x}(t)$ is unstable and thus quickly collapses onto $x(t)$. This occurs both in one dimension as well as in higher-dimensional systems [26]. Given that instabilities destroy the mechanisms of current suppression due to the contributions of a trajectory and the transformed one, the observed suppression of directed transport in onedimensional systems must be associated to a different mechanism. This suppression under antisymmetric forces is actually a consequence of the symmetry (7), which yields no current for systems - which include spatially symmetric as well as spatially shift-symmetric systems of interest here-satisfying the property (4)

$$
\begin{aligned}
v[f(x)+F(t)] & =-v[f(x)-F(t)]=-v[f(x)+F(-t)] \\
& =-v[f(x)+F(t)] .
\end{aligned}
$$

A consequence of this analysis is that truly spatially symmetric systems should also exhibit no current in one-dimensional overdamped systems when antisymmetric forces are driving the system. This phenomenon is illustrated in Fig. 3. It was already experimentally observed in Ref. [45], but it remained unexplained until the present Letter. These results are a confirmation of the validity of the approach based on a more general symmetry analysis that does not rely on the direct analysis of the solutions of the equation of motion.

It is worth stressing that in the present discussion the dimensionality of the system corresponds to the number of spatial degrees of freedom taking part into the rectification mechanism, and not necessarily to the dimensionality of the potential landscape. The violation of the symmetries (6), (7) in the above 2D overdamped setup is due to a rectification mechanism taking place in the two perpendicular directions. However, the symmetries (6), (7) are not restricted to strictly one-dimensional systems; they are still present in higher-dimensional overdamped systems provided that the rectification mechanism involves one spatial dimension only. For example, the dashed line in Fig. 3(c) shows the suppression of current for antisymmetric driving for the same 2D system shown in Fig. 2 when the biharmonic driving force is applied in the $y$ direction only. Additional examples are shown in [26].
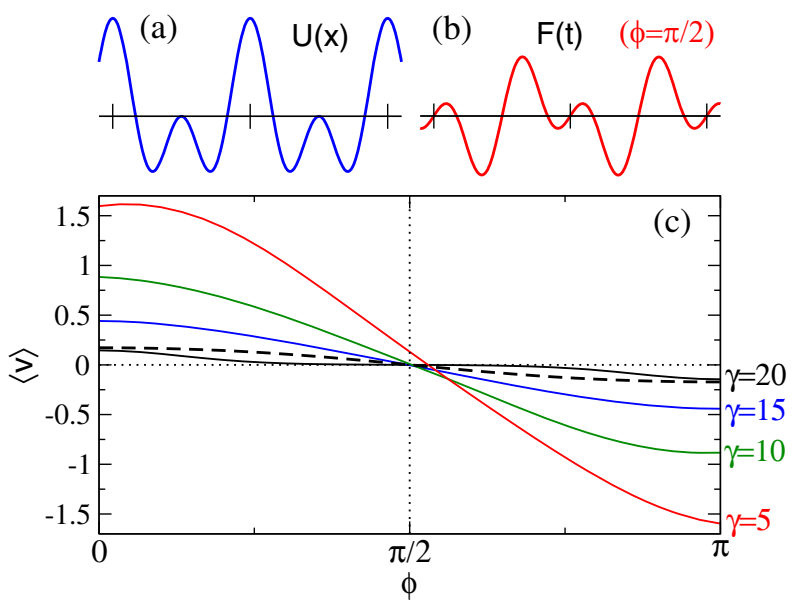

FIG. 3. Current suppression in one-dimensional overdamped systems with a spatially symmetric potential and applied antisymmetric forces. The driving force has a biharmonic shape, $F(t)=A[\cos (\omega t)+\cos (2 \omega t+\phi)]$. (a) Spatially symmetric potential $U(x)=U_{0}[\cos (k x)+\cos (2 k x)]$. (b) The driving force $F(t)$ is antisymmetric when $\phi=\pi / 2$. (c) Directed current as a function of the driving phase $\phi$, for different levels of damping. Reduced units are defined such that $m=k=\omega=1$. Other parameters are $U_{0}=20$ and $A=\Gamma=40$. The dashed line shows, for comparison, the current $\left(\left\langle v_{y}\right\rangle\right)$ for the same driving force applied in the $y$ direction and a two-dimensional potential $U(x, y)=U_{0} \cos (k x)[1+\cos (4 k y)]$ in the overdamped regime $\left(\gamma=U_{0}=50, A=2 \gamma, \Gamma=0.1 \gamma^{2}\right)$. 
Discussion.-The hidden symmetries identified in the present Letter are of relevance to current experiments, and they also allow us to recast known results within a more general theoretical framework. This is well exemplified by the two specific case studies that are presented below.

The first case study corresponds to the system of ac-driven vortices trapped in a superconductor that was experimentally studied in Ref. [14]. Here, interparticle interactions provide an additional path to escape from the symmetries (6), (7). Our results of Fig. 4 refer precisely to the one-dimensional system of interacting Brownian particles that was successfully used in Ref. [14] to explain the multiple current reversals observed on ac-driven vortices trapped in a superconductor. Despite not being strictly satisfied, the influence of the symmetries (6), (7) is quite noticeable, canceling the ratchet effect and most of the current reversals in regions of the parameter space where the appearance of a current is not directly related to particle interactions.

For a second case study, we refer to the celebrated flashing ratchet model $[8-11,46,47]$, where the ratchet potential is periodically switched on and off in the absence of any additional additive driving $F(t)$-i.e., here $\mathcal{F}(x, t)=-\partial U(x, t) / \partial x$ - and more specifically to the known result [48] that a flashing shift-symmetric potential cannot produce directed motion. The theoretical framework and the related new symmetries that we introduce here allow for a simple explanation of such a result.

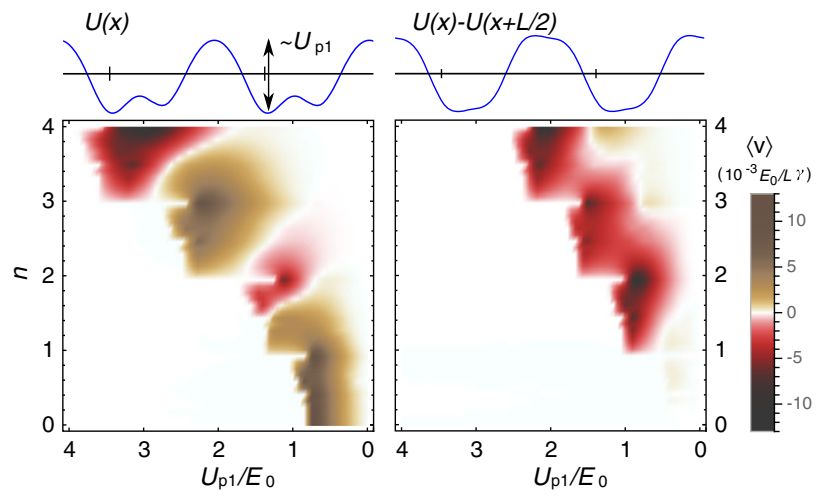

FIG. 4. Cancellation of transport, via the use of shift-symmetric potentials, for the one-dimensional overdamped system of interacting particles from Ref. [14]. The bottom panels show the net chain current as a function of the number of particles per period, $n$, and the potential depth $U_{p 1} / E_{0}$, with the left panels referring to the original one-particle potential $U(x)$ (depicted in the upper panel), and the right panels to a shift-symmetric potential built from the former as $U_{\mathrm{ss}}(x)=U(x)-U(x+L / 2)$. The interaction between the particles is accounted for by the pair potential $V_{\text {int }}(r)=-E_{0} \ln (r)$, with $r$ the particle separation. The system is driven by a single-harmonic force acting on each particle, which is both shift symmetric and antisymmetric. The parameters are the same as in Fig. 2 of [14].
In one-dimensional overdamped systems, the following symmetry is generally satisfied [26]:

$$
v[\mathcal{F}(-x,-t)]=v[\mathcal{F}(x, t)] .
$$

In two-state systems that are periodically switched, reversing the direction of time has no effect, $v[\mathcal{F}(x,-t)]=v[\mathcal{F}(x, t)]$; this fact, together with (2), (10), and (3), yields no current for shift-symmetric potentials,

$$
\begin{aligned}
v[\mathcal{F}(x, t)] & =-v[-\mathcal{F}(-x, t)]=-v[-\mathcal{F}(x,-t)] \\
& =-v[-\mathcal{F}(x, t)]=-v[-\mathcal{F}(x+L / 2, t)] \\
& =-v[\mathcal{F}(x, t)] .
\end{aligned}
$$

Therefore, a flashing ratchet with a shift-symmetric potential, regardless of whether it is spatially asymmetric, cannot produce directed motion; this thus shows that in overdamped systems shift-symmetric potentials behave like spatially symmetric ones.

Conclusions.-The present Letter addresses the outstanding issue of providing a general theoretical framework for the identification of symmetries not captured by the standard symmetry analysis, examples of which were already given in previous works [44,48] with ad hoc treatments. We have proven the existence in a prototypical 1D overdamped system of hidden symmetries, which escape identification by the standard symmetry analysis and require different theoretical tools for their revelation. Though not rigorously satisfied in higher-dimensional systems, the effects of hidden symmetries have been shown to be still noticeable in them. Our results pave the way for new mechanisms of manipulating transport. In fact, the hidden symmetries determine current reversals, which can be used to precisely control transport and implement mechanisms for particle separation. Specific realizations for optical tweezers and cold atom setups are discussed in the Supplemental Material [26].

Financial support from the Royal Society (Grant No. IE130734) (D. C. and F. R.), and the Leverhulme Trust (Grant No. RPG 2012 809) (F. R.) is acknowledged.

*dcubero@us.es

f.renzoni@ucl.ac.uk

[1] R. D. Astumian, Thermodynamics and kinetics of a Brownian motor, Science 276, 917 (2000).

[2] P. Reimann, Brownian motors: Noisy transport far from equilibrium, Phys. Rep. 361, 57 (2002).

[3] P. Hänggi and F. Marchesoni, Artificial Brownian motors: Controlling transport on the nanoscale, Rev. Mod. Phys. 81, 387 (2009).

[4] L. Mahadevan and P. Matsudaira, Motility powered by supramolecular springs and ratchets, Science 288, 95 (2000). 
[5] M. Schliwa and G. Woehlke, Molecular motors, Nature (London) 422, 759 (2003).

[6] J. V. Hernández, E. R. Kay, and D. A. Leigh, A reversible synthetic rotary molecular motor, Science 306, 1532 (2004).

[7] M. V. Costache and S. O. Valenzuela, Experimental spin ratchet, Science 330, 1645 (2010).

[8] J. Rousselet, L. Salome, A. Ajdari, and J. Prost, Directional motion of Brownian particles induced by a periodic asymmetric potential, Nature (London) 370, 446 (1994).

[9] J. S. Bader, R. W. Hammond, S. A. Henck, M. W. Deem, G. A. McDermott, J. M. Bustillo, J. W. Simpson, G. T. Mulhern, and J. M. Rothberg, DNA transport by a micromachined Brownian ratchet device, Proc. Natl. Acad. Sci. U.S.A. 96, 13165 (1999).

[10] C. F. Chou, O. Bakajin, S. W. P. Turner, T. A. J. Duke, S. S. Chan, E. C. Cox, H. G. Craighead, and R. Austin, Sorting by diffusion: An asymmetric obstacle course for continuous molecular separation, Proc. Natl. Acad. Sci. U.S.A. 96, 13762 (1999).

[11] A. van Oudenaarden and S. G. Boxer, Brownian ratchets: Molecular separations in lipid bilayers supported on patterned arrays, Science 285, 1046 (1999).

[12] S. Matthias and F. Müller, Asymmetric pores in a silicon membrane acting as massively parallel brownian ratchets, Nature (London) 424, 53 (2003).

[13] J. E. Villegas, S. Savel'ev, F. Nori, E. M. Gonzalez, J. V. Anguita, R. Garca, and J.L. Vicent, A superconducting reversible rectifier that controls the motion of magnetic flux quanta, Science 302, 1188 (2003).

[14] C. C. de Souza Silva, J. Van de Vondel, M. Morelle, and V. V. Moshchalkov, Controlled multiple reversals of a ratchet effect, Nature (London) 440, 651 (2006).

[15] H. Linke, T. E. Humphrey, A. Löfgren, A. O. Sushkov, R. Newbury, R. P. Taylor, and P. Omling, Experimental tunneling ratchets, Science 286, 2314 (1999).

[16] T. Salger, S. Kling, T. Hecking, C. Geckeler, L. MoralesMolina, and M. Weitz, Directed transport of atoms in a Hamiltonian quantum ratchet, Science 326, 1241 (2009).

[17] C. Drexler, S. A. Tarasenko, P. Olbrich, J. Karch, M. Hirmer, F. Müller, M. Gmitra, J. Fabian, R. Yakimova, S. Lara-Avila, S. Kubatkin, M. Wang, R. Vajtai, P. M. Ajayan, J. Kono, and S. D. Ganichev, Magnetic quantum ratchet effect in graphene, Nat. Nanotechnol. 8, 104 (2013).

[18] V. Serreli, C. F. Lee, E. R. Kay, and D. A. Leigh, A molecular information ratchet, Nature (London) 445, 523 (2007).

[19] T. R. Kelly, H. de Silva, and R. A. Silva, Undirectional rotary motion in a molecular system, Nature (London) 401, 150 (1999).

[20] J. Siegel, Inventing the nanomolecular wheel, Science 310, 63 (2005).

[21] R. Gommers, S. Bergamini, and F. Renzoni, DissipationInduced Symmetry Breaking in a Driven Optical Lattice, Phys. Rev. Lett. 95, 073003 (2005).

[22] S. Flach, O. Yevtushenko, and Y. Zolotaryuk, Directed Current Due to Broken Time-Space Symmetry, Phys. Rev. Lett. 84, 2358 (2000).

[23] S. Denisov, S. Flach, and P. Hänggi, Tunable transport with broken spacetime symmetries, Phys. Rep. 538, 77 (2014).
[24] W. Thomson (Lord Kelvin), The kinetic theory of the dissipation of energy, Proc. Roy. Soc. Edinburgh 8, 325 (1874).

[25] J. Casado-Pascual, J. A. Cuesta, N. R. Quintero, and R. Alvarez-Nodarse, General approach for dealing with dynamical systems with spatiotemporal periodicities, Phys. Rev. E 91, 022905 (2015).

[26] See Supplemental Material at http://link.aps.org/ supplemental/10.1103/PhysRevLett.116.010602, which includes Refs. [27-40], for a proof of Eqs. (6) and (7), a discussion on the role of instabilities in a $2 \mathrm{D}$ overdamped system, and on the control of transport at the nanoscale about the symmetry points identified in the present work.

[27] H. Risken, The Fokker-Planck Equation (Springer, Berlin, 1984).

[28] L. P. Faucheux, L. S. Bourdieu, P. D. Kaplan, and A. J. Libchaber, Optical Thermal Ratchet, Phys. Rev. Lett. 74, 1504 (1995).

[29] S.-H. Lee, K. Ladavac, M. Polin, and D. G. Grier, Observation of Flux Reversal in a Symmetric Optical Thermal Ratchet, Phys. Rev. Lett. 94, 110601 (2005).

[30] A. V. Arzola, K. Volke-Sepulveda, and J. L. Mateos, Experimental Control of Transport and Current Reversals in a Deterministic Optical Rocking Ratchet, Phys. Rev. Lett. 106, 168104 (2011).

[31] O. M. Marago, P. H. Jones, P. G. Gucciardi, G. Volpe, and A. C. Ferrari, Optical trapping and manipulation of nanostructures, Nat. Nanotechnol. 8, 807 (2013).

[32] C.-S. Lee, B. Jankó, I. Derényi, and A.-L. Barabási, Reducing vortex density in superconductors using the 'ratchet effect', Nature (London) 400, 337 (1999).

[33] D. E. Shalóm and H. Pastoriza, Vortex Motion Rectification in Josephson Junction Arrays with a Ratchet Potential, Phys. Rev. Lett. 94, 177001 (2005).

[34] J. F. Wambaugh, C. Reichhardt, C. J. Olson, F. Marchesoni, and F. Nori, Superconducting Fluxon Pumps and Lenses, Phys. Rev. Lett. 83, 5106 (1999).

[35] D. Cole, S. Bending, S. Savel'ev, A. Grigorenko, T. Tamegai, and F. Nori, Ratchet without spatial asymmetry for controlling the motion of magnetic flux quanta using time-asymmetric drives, Nat. Mater. 5, 305 (2006).

[36] C. Mennerat-Robilliard, D. Lucas, S. Guibal, J. Tabosa, C. Jurczak, J.-Y. Courtois, and G. Grynberg, Ratchet for Cold Rubidium Atoms: The Asymmetric Optical Lattice, Phys. Rev. Lett. 82, 851 (1999).

[37] M. Schiavoni, L. Sanchez-Palencia, F. Renzoni, and G. Grynberg, Phase Control of Directed Diffusion in a Symmetric Optical Lattice, Phys. Rev. Lett. 90, 094101 (2003).

[38] R. Gommers, S. Denisov, and F. Renzoni, Quasiperiodically Driven Ratchets for Cold Atoms, Phys. Rev. Lett. 96, 240604 (2006).

[39] D. Cubero, V. Lebedev, and F. Renzoni, Current reversals in a rocking ratchet: Dynamical vs symmetry-breaking mechanisms, Phys. Rev. E 82, 041116 (2010).

[40] A. Wickenbrock, P. C. Holz, N. A. Abdul Wahab, P. Phoonthong, D. Cubero, and F. Renzoni, Vibrational Mechanics in an Optical Lattice: Controlling Transport via Potential Renormalization, Phys. Rev. Lett. 108, 020603 (2012). 
[41] P. Reimann, Supersymmetric Ratchets, Phys. Rev. Lett. 86, 4992 (2001).

[42] S. Denisov, Y. Zolotaryuk, S. Flach, and O. Yevtushenko, Vortex and Translational Currents due to Broken TimeSpace Symmetries. Phys. Rev. Lett. 100, 224102 (2008).

[43] D. Speer, Ph.D. thesis, Universität Bielefeld, 2011.

[44] S. Denisov, S. Flach, A. A. Ovchinnikov, O. Yevtushenko, and Y. Zolotaryuk, Broken space-time symmetries and mechanisms of rectification of ac fields by nonlinear (non)adiabatic response, Phys. Rev. E 66, 041104 (2002).
[45] S. Ooi, S. Savel'ev, M. B. Gaifullin, T. Mochiku, K. Hirata, and F. Nori, Nonlinear Nanodevices Using Magnetic Flux Quanta, Phys. Rev. Lett. 99, 207003 (2007).

[46] A. Ajdari and J. Prost, Mouvement induit par un potentiel périodique de basse symmétrie: Diélectrophorèse pulsée, C.R. Acad. Sci. Paris II 315, 1635 (1992).

[47] G. P. Harmer and D. Abbott, Losing strategies can win by Parrondo's paradox, Nature (London) 402, 864 (1999).

[48] R. Kanada and K. Sasaki, Thermal ratchets with symmetric potentials, J. Phys. Soc. Jpn. 68, 3759 (1999). 